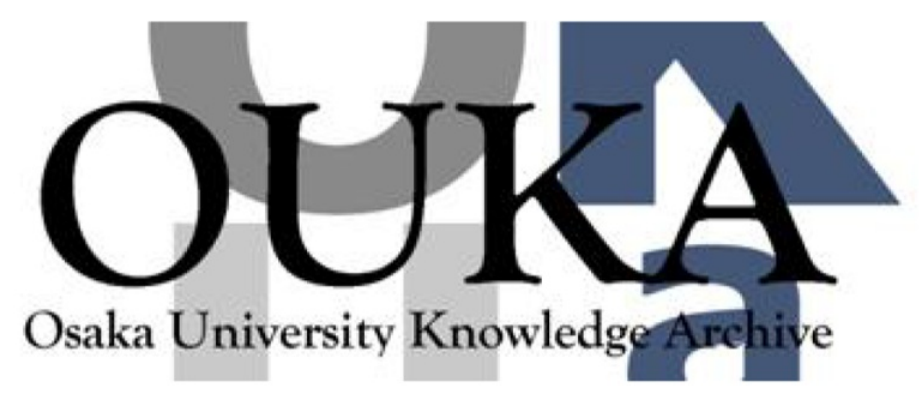

\begin{tabular}{|c|l|}
\hline Title & $\begin{array}{l}\text { Resonance techniques and apparatus for elastic- } \\
\text { wave velocity determination in thin metal } \\
\text { plates }\end{array}$ \\
\hline Author(s) & $\begin{array}{l}\text { Petersen, G. L. ; Chick, B. B. ; Fortunko, C. M. et } \\
\text { al. }\end{array}$ \\
\hline Citation & $\begin{array}{l}\text { Review of Scientific Instruments. 65(1) p. 192- } \\
\text { p. 198 }\end{array}$ \\
\hline Issue Date & $1994-01$ \\
\hline oaire:version & VoR \\
\hline URL & https://hdl. handle. net/11094/3455 \\
\hline rights & \\
\hline Note & \\
\hline
\end{tabular}

Osaka University Knowledge Archive : OUKA

https://ir. Library. osaka-u. ac. jp/

Osaka University 


\section{AIP Scientific Instruments}

\section{Resonance techniques and apparatus for elasticwave velocity determination in thin metal plates}

G. L. Petersen, B. B. Chick, C. M. Fortunko, and M. Hirao

Citation: Rev. Sci. Instrum. 65, 192 (1994); doi: 10.1063/1.1144776

View online: http://dx.doi.org/10.1063/1.1144776

View Table of Contents: http://rsi.aip.org/resource/1/RSINAK/v65/i1

Published by the American Institute of Physics.

\section{Related Articles}

Porous medium acoustics of wave-induced vorticity diffusion Appl. Phys. Lett. 98, 084101 (2011)

Second-harmonic generation accompanying nonlinear reflection of shear waves in an isotropic layered structure J. Appl. Phys. 82, 1026 (1997)

Wave propagation in a piezoelectric layer

J. Appl. Phys. 81, 7242 (1997)

Determination of the viscous and thermal characteristic lengths of plastic foams by ultrasonic measurements in helium and air

J. Appl. Phys. 80, 2009 (1996)

A comparison between wave propagation in watersaturated and airsaturated porous materials J. Appl. Phys. 73, 28 (1993)

\section{Additional information on Rev. Sci. Instrum.}

Journal Homepage: http://rsi.aip.org

Journal Information: http://rsi.aip.org/about/about_the_journal

Top downloads: http://rsi.aip.org/features/most_downloaded

Information for Authors: http://rsi.aip.org/authors

\section{ADVERTISEMENT}

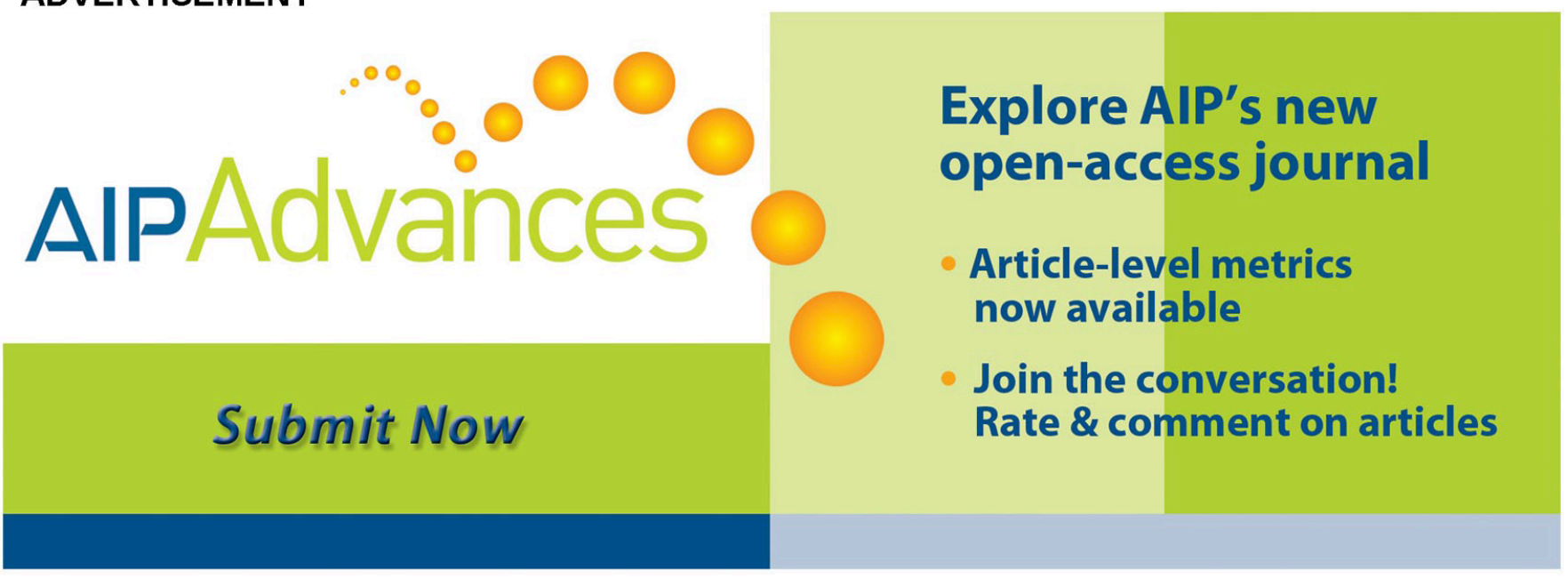




\title{
Resonance techniques and apparatus for elastic-wave velocity determination in thin metal plates
}

\author{
G. L. Petersen and B. B. Chick \\ Ritec, Inc., 60 Alhambra Road, Suite 5, Warwick, Rhode Island 02886 \\ C. M. Fortunko \\ National Institute of Standards and Technology, Materials Reliability Division, 325 S. Broadway, \\ Boulder, Colorado 80303 \\ M. Hirao \\ Faculty of Engineering Science, Osaka University, Toyonaka, Osaka 560, Japan
}

(Received 16 November 1992; accepted for publication 1 September 1993)

\begin{abstract}
The principles of operation of a new pulsed ultrasonic resonance spectrometer are presented. The system incorporates: (1) the use of electromagnetic-acoustic transducers (EMATs), (2) a method of obtaining acoustic signals similar to the sampled continuous-wave scheme described by Bolef and Miller, (3) the Clark method of recording line shapes, and (4) a high power gated rf amplifier and a unique implementation of superheterodyne and quadrature phase sensitive detection circuitry. The result is a spectrometer capable of measuring both shear and longitudinal ultrasonic velocities in very thin samples with much greater reproducibility than other pulse methods. It also has applicability in mcasuring attenuation and other physical properties such as composite material bonding that can be accessed using ultrasound. Experimental results of tests using EMATs in the $0.1-20 \mathrm{MHz}$ range on thin (1.27 and $2.49 \mathrm{~mm}$ thick) aluminum sheets are presented.
\end{abstract}

\section{INTRODUCTION}

There is considerable interest in improving the accuracy of existing ultrasonic velocity measurement methods and devising new ones in response to new measurement needs. New measurement-system requirements continually arise as a result of developments in science and technology. For example, recent advances in high- $T_{c}$ superconductivity have generated an interest in accurate determination of elastic-wave velocities, both shear and compressional, in very small samples. ${ }^{1}$ Samples with dimensions of the order of $1 \mathrm{~mm}$ and smaller are not uncommon. ${ }^{2}$ Also, new developments in on-line process control (measurement of metallurgical texture) have resulted in a requirement for systems capable of absolute sound-speed determination in metal sheets whose thicknesses range from 1 to $2 \mathrm{~mm}^{3}$

Velocity measurements using traditional time-domain methods are often difficult, if not impossible, to make in small samples and thin sheets, primarily because of transducer bandwidth and center-frequency limitations. However, various resonance techniques, all of which are fundamentally frequency-domain methods, can be very useful in such cases.

The many experimental difficulties of sound-speed determination in small samples have been recognized for some time. A number of the major difficulties were resolved by McSkimmin who developed the "long pulse" method. ${ }^{2}$ Others were addressed later by Bolef and Miller, and by Breazeale et al., who investigated the use of continuous-wave (cw) methods and developed the sampled cw technique. ${ }^{3-6}$ More recently, Pialucha et al. described an amplitude-spectrum technique to measure the compressional-wave phase velocity in thin specimens. ${ }^{7}$

To achieve high absolute accuracy, the methods of
Bolef and Miller rely on weak mechanical loading of the specimen by the transducers. Equivalently, the method of Pialucha et al. relies on a large acoustic-impedance mismatch between the specimen and the liquid used to couple the ultrasonic probing signals. In both methods, the elasticwave velocities are extracted using the frequency-domain response maxima or minima. It is often of interest to determine the velocities in thin plate geometries in which several elastic-wave modes can propagate. A measurement method for accomplishing this has been described by Maze and co-workers. ${ }^{8}$

In the past, piezoelectric transducers were generally used to generate and detect the ultrasonic signals. Such transducers are efficient and can operate over a large frequency range. However, they must operate in intimate contact with the specimen. As a result, they may not be suitable for many resonance measurements. To overcome this limitation, we prefer to use noncontacting, weakly coupled transducers. In particular, this article describes the operation of a measurement system that uses electromagneticacoustic transducers (EMATs). EMATs do not require intimate contact and can operate in the high megahertz frequency regions. Resonant frequencies as high as 120 $\mathrm{MHz}$ have been reported by Kawashima and Wright. ${ }^{9}$

The intrinsic benefits of weak coupling between transducers and specimens are widely recognized. Therefore, there has long been an interest in the use of various noncontact ultrasonic transduction mechanisms. For example, Kondratev demonstrated the use of broadband (1-70 MHz) electrostatic ultrasonic transducers (ESATs) to make very accurate $\mathrm{cw}$ measurements of compressionalwave velocity and attention along the through-thickness direction in thin metal specimens. ${ }^{10}$ Air coupled transduc- 
ers operating well below $1 \mathrm{MHz}$ were used by Luukkala to investigate the dispersion characteristics of plate waves in paper and metal sheets. ${ }^{11,12}$ Recently, EMATs operating at $0.5 \mathrm{MHz}$ were used to map metallurgical textures in steel sheets. ${ }^{13}$

The EMATs were selected for this work because they can be designed to generate and detect both longitudinally and transversely polarized elastic waves in thin metal plates. This capability was needed to provide an alternative technique for determining metallurgical textures in steel sheets. Also, EMATs are inherently compatible with the use of sampled cw measurement techniques.

\section{MEASUREMENT SYSTEM CONCEPT}

Our measurement system is based on the sampled $\mathrm{cw}$ techniques of Bolef and Miller. Sampled cw techniques typically involve the use of a low-level $\mathrm{cw}$ driving signal. The main advantage of the long pulse and sampled $\mathrm{cw}$ techniques is that the elastic-wave displacements within the specimen can be gradually increased to a nearequilibrium value which is generally determined by the magnitude of loss mechanisms within the specimen and external loading by transducers, without exceeding the breakdown limits of the transducers. When the electrical driving signals are abruptly interrupted, the elastic-wave displacements begin to decay. The transient elastic-wave signals, which follow the abrupt interruption of the driving signals, are then processed using an appropriate algorithm to determine the elastic-wave velocities. Elastic-wave velocities are calculated using sample dimensions and resonant frequencies of several resonances corresponding to the same elastic-wave mode.

Although our measurement approach is conceptually similar to the sampled $\mathrm{cw}$ method, we take advantage of several advances in instrumentation and signal processing to improve the absolute accuracy of the measurement. Specifically, we employ a very high-power gated rf amplifier to drive a shear-wave EMAT and use a phase-sensitive, gated analog integrator as a spectrometer to process the received transient ultrasonic signals. This method of using analog integration to obtain spectroscopic data is not new and has been reported previously by Clark. ${ }^{14}$ The application of the "Clark method" to the study of nuclear magnetic resonance has been investigated in considerable detail by Avogadro et al. ${ }^{15}$

Analog spectroscopic methods, such as the Clark method, have been largely replaced by digital methods, such as those employing the fast Fourier transform (FFT) algorithm. However, analog spectroscopic methods offer significant advantages in certain applications. For example, standard digital FFT signal processing requires that the bandwidth of the driving signal be larger than the linewidth of the resonance. This means that short driving pulses or rf bursts are needed, and the amount of energy available to buildup a strong resonant standing wave is limited.

Figure 1 shows a simplified block diagram of our apparatus, including the experimental configuration. The noncontacting EMAT transducer used in this configura-

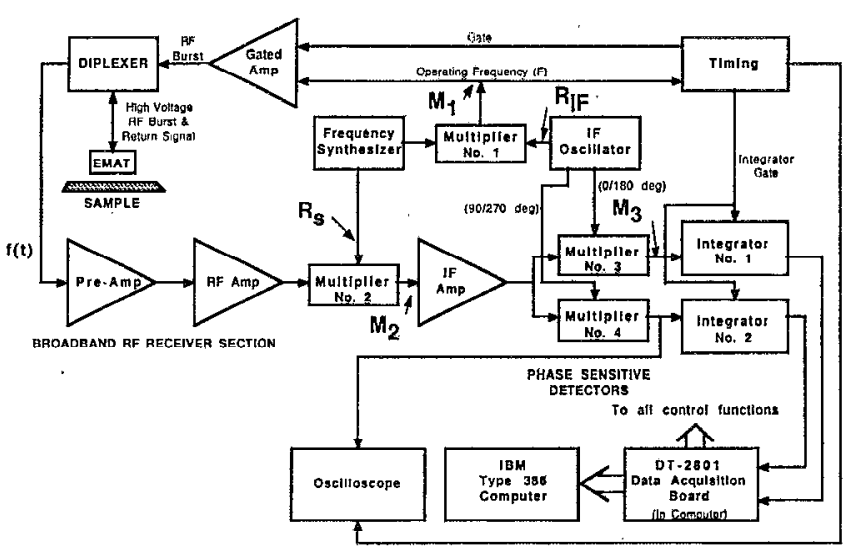

FIG. 1. Simplified block diagram of the acoustic resonance apparatus.

tion generates and detects shear waves that propagate along the through-thickness direction of the plate. If the plate exhibits elastic birefringence, then two distinct shearwave signals, propagating at slightly different velocities, can be observed.

In the configuration of Fig. 1, the EMAT is driven with a long burst of $\mathrm{rf}$, and the same transducer examines the acoustic ringing in the sample. Figure 2 represents the time-domain appearance of the received ultrasonic signals as well as the gating wave form for the integrators. The gating interval commences after a very short time delay (a few microseconds) following the interruption of the driving rf burst.

\section{APPARATUS AND SIGNAL PROCESSING}

\section{A. Transmitter electronics}

A narrow band $\mathrm{rf}$ burst is produced from a synthesized continuous-wave (cw) signal using a high power gated amplifier capable of producing $1 \mathrm{kV}$ peak-to-peak rf bursts into $50 \Omega$. The rf burst is applied to a shear wave EMAT, whose construction is illustrated conceptually in Fig. 3. The rf burst is chosen with sufficient length (up to $200 \mu \mathrm{s}$ ) to buildup strong ultrasonic standing-wave signals within the sample. In practice, the rf burst length may not be long enough to establish steady-state, near-equilibrium resonance conditions. However, typical rf burst lengths are sufficient to establish favorable signal-to-noise conditions

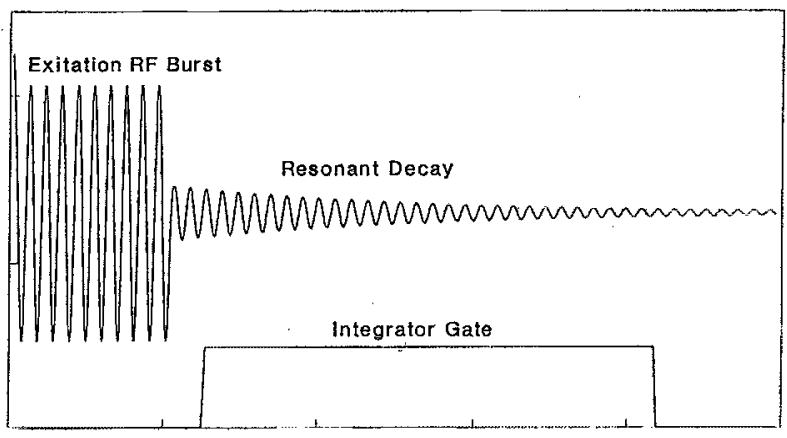

FIG. 2. Diagrammatic representation of the driving $\mathrm{rf}$ burst and received rf resonant acoustic ringing. 


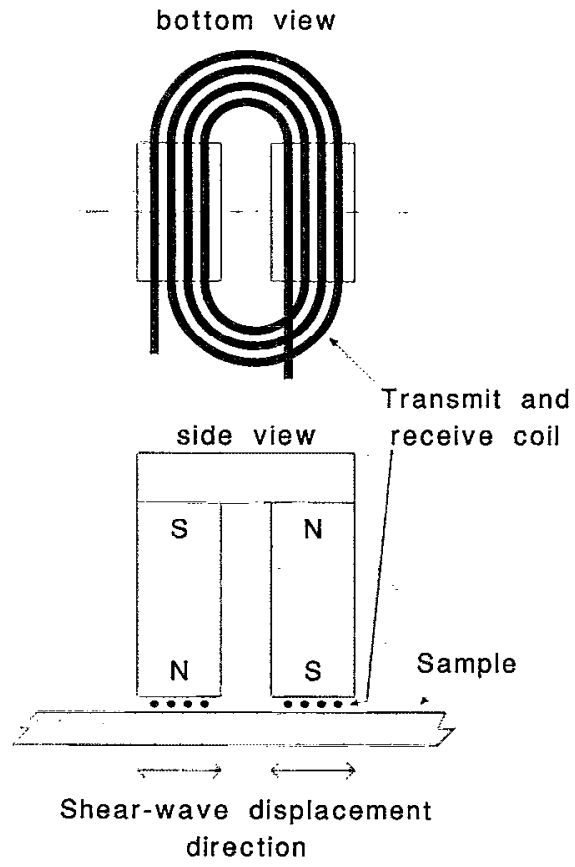

FIG. 3. Diagrammatic representation of a shear-wave EMAT showing the spatial relationship between the coil and permanent magnets.

in most engineering materials. The amplitudes of the resonant ultrasonic fields are directly proportional to the voltage of the driving pulse and increase monotonically with $\mathrm{rf}$ burst length. In low-loss materials the ultrasonic amplitudes approach equilibrium slowly, but become quite high. As a consequence, long $\mathrm{rf}$ bursts are not needed. In lossy materials, even though equilibrium levels are small in amplitude they are achieved quickly, and, therefore, long rf bursts also are not required.

Figure 1 illustrates the block diagram of our experimental approach. Currently, the apparatus may operate in the $100 \mathrm{kHz}-20 \mathrm{MHz}$ region. The cw driving signal is not generated directly by the synthesizer, but is instead obtained by using analog Multiplier No. 1 to multiply the reference output of the intermediate frequency (IF) oscillator

$$
R_{\mathrm{IF}}=A_{\mathrm{IF}} \cos \left(2 \pi I F t+\theta_{\mathrm{IF}}\right),
$$

by the output of a higher frequency synthesizer

$$
R_{S}=A_{S} \cos \left[2 \pi(\mathrm{IF}+F) t+\theta_{s}\right] .
$$

After filtering, the multiplication produces the operating frequency reference signal.

$$
M_{1}=A_{F} \cos \left(2 \pi F t+\theta_{s}-\theta_{\mathrm{IF}}\right)+\text { High Freq. Term. }
$$

In Eq. (3), the terms $\theta_{\mathrm{IF}}$ and $\theta_{s}$ refer to the arbitrary phases of the IF ( $25 \mathrm{MHz}$ ) oscillator and the synthesizer, and $A_{F}$ is the amplitude of the $M_{1}$ output. The multiplication produces difference and sum frequencies. The sumfrequency term, $(2 \mathrm{IF}+F)$, is first removed using a lowpass filter at the output of the analog multiplier. The difference-frequency term is then applied to the input of the gated amplifier, which produces the high-power, rfburst electrical signals needed to drive the EMAT.

\section{B. Diplexer and preamplifier electronics}

In the experimental configuration of Fig. 1, the transmitting EMAT is also used to detect the ultrasonic signals reverberating within the thin-sheet specimen. To protect the sensitive receiver-preamplifier circuitry from being damaged by the high-voltage transmitter signals, we use a transformer coupled diplexer circuit. The diplexer routes the high-level transmitter current to the EMAT during the transmit cycle, and routes the low-level received signal to the preamplifier, with minimal attenuation during the receive cycle. The effective bandwidth of our front-end electronics, including the transmitter, diplexer, and preamplifier circuits, exceeds $20 \mathrm{MHz}$.

\section{Superheterodyne receiver and phase-sensitive detector}

Our apparatus uses a superheterodyne receiver equipped with a phase-sensitive detector to process the received ultrasonic signals following wideband preamplification. The first step of superheterodyne reception involves a multiplication operation that preserves the phase relationships of the received ultrasonic signals while shifting their carrier frequencies to an intermediate-frequency (IF) band. The frequency shifting is accomplished using an analog rf multiplier circuit. Following amplification and filtering by the IF amplifier, the ultrasonic signals are shifted again to zero rf frequency using a pair of matched if multiplier circuits. The two multipliers are driven by separate cw reference signals at the IF frequency. The two IF reference signals are at exactly the same frequency, but are shifted by 90 electrical degrees. Following multiplication by the two IF reference signals, the ultrasonic signals are filtered by low-pass filters and integrated by matched gated integrators. The outputs of the integrators are sampled and converted to digital format using a 12-bit analog-to-digital (A/D) converter. Further processing, needed to determine the resonance frequencies of a thin sheet, is accomplished by computer.

To gain more insight into the operation of the phasesensitive receiver circuitry, it is instructive to represent the received ultrasonic signals as a narrow-band signal. Such a signal can be represented as a monochromatic signal at frequency $\left(F_{r}\right)$ modulated by a slowly varying amplitude function of time $A_{r}(t)$. Physically, $A_{r}(t)$ is a monotonic function that describes the decay of the ultrasonic signals within the specimen following the interruption of the driving rf electrical burst. In our implementation of the heterodyne receiver, the signal $f(t)$ is amplified by the variable-gain (78- $\mathrm{dB}$ range) wideband if amplifier. The output of the wideband amplifier is then multiplied by the synthesizer output signal $R_{s}$ at frequency $(F+\mathrm{IF})$ using the analog multiplier 2 . The multiplication results in a new signal with a frequency IF. Although the ultrasonic signals received following the interruption of the driving $\mathrm{rf}$ signals are generally at a frequency different from the transmitted 
frequency $F$ they retain the phase terms $\theta_{\mathrm{IF}}$ and $\theta_{s}$ shown in Eq. (3). Assuming that the frequency of the received signal is $F_{r}$, the signal $f(t)$, appearing at the input of the pre-amplifier can be represented using the general form

$$
f(t)=A_{r}(t) \cos \left(2 \pi F_{r} t+\theta_{s}-\theta_{\mathrm{IF}}+\theta_{r}\right),
$$

where $\theta_{r}$ represents the phase shifts in the transducer and sample, and the other phase shift terms follow from Eq. (3). When $f(t)$ after amplification is multiplied by the synthesizer output in multiplier 2 the resulting voltage is

$$
\begin{aligned}
M_{2}= & g_{2} A_{r}(t) \cos \left\{2 \pi\left[\mathrm{IF}+\left(F-F_{r}\right)\right] t+\theta_{\mathrm{IF}}-\theta_{r}\right\} \\
& + \text { High Freq. Term, }
\end{aligned}
$$

where the constant $g_{2}$ includes the rf gain, the amplitude of the reference $R_{s}$, and the conversion efficiency of the multiplier. The high frequency term of Eq. (5) involving the frequencies (IF $+F+F_{r}$ ) is completely rejected by the IF amplifier, which has a fixed center frequency of $25 \mathrm{MHz}$ and three computer-settable bandwidths: $0.4,1$, and 4 $\mathrm{MHz}$. The remaining signal, containing the phase of the ultrasonic signal, is amplified and multiplied by the IF oscillator outputs using analog multipliers 3 and 4 . The output of multiplier 3 is

$M_{3}=A_{r}(t) g_{3} \cos \left[2 \pi\left(F-F_{r}\right) t-\theta_{r}\right]+$ High Freq. Term,

where the total gain and conversion efficiencies are all included in the term $g_{3}$. The output of multiplier 4 is

$M_{4}=A_{r}(t) g_{3} \sin \left[2 \pi\left(F-F_{r}\right) t-\theta_{r}\right]+$ High Freq. Term.

Equations (5), (6), and (7) describe the quadrature phase-sensitive detection. The high-frequency terms are effectively removed by low-pass filters (not shown in Fig. 1) that follow analog multipliers 3 and 4 . Only the lowfrequency (base band) terms, involving the difference between the driving and the received frequencies $\left(F-F_{r}\right)$ survive. The phase terms $\theta_{\mathrm{IF}}$ and $\theta_{s}$ are also removed.

\section{Analog integration}

The output of multiplier 3 can be described by simply multiplying the received ultrasonic signal $f(t)$ [Eq. (4)] by the operating frequency reference $M_{1}$ [Eq. (3)] and a constant $k$ which includes the gain and other constant factors. An expression for $M_{4}$ can be obtained by a similar process and, therefore, the outputs of the gated analog integrators may be expressed by

Integrator No. 1 Output

$$
=k \int_{T_{1}}^{T_{2}} f(t) \cos \left(2 \pi F t+\theta_{S}+\theta_{\mathrm{IF}}\right) d t
$$

Integrator No. 2 Output

$$
=k \int_{T_{1}}^{T_{2}} f(t) \sin \left(2 \pi F t+\theta_{S}-\theta_{\mathrm{IF}}\right) d t,
$$

where $T_{1}$ typically corresponds to time at which the driving rf signals are interrupted plus the several microseconds required for the receiver circuitry to settle. The upper limit $T_{2}$ typically extends beyond the region where the received signals become unobservable.

In our measurement method, the spectra of the received ultrasonic signals are determined by incrementally stepping the frequency $(F-I F)$ through a range of interest and recording the voltages of the integrator outputs at each frequency step. This is essentially the Clark method.

The integral expressions in Eqs. (8) and (9) closely resemble the cosine $[C(F)]$ and sine $[S(F)]$ parts of a Fourier transform $[G(F)]$ as defined in Eq. (10)

$$
G(F)=\frac{1}{2 \pi} \int_{-\infty}^{\infty} f(t)[\cos (2 \pi F t)-i \sin (2 \pi F t)] d t .
$$

We do not normally display the sine and cosine transforms individually. Instead we calculate and display the amplitude spcetrum as given by

$$
\operatorname{Amp}(F)=\left[C(F)^{2}+S(F)^{2}\right]^{1 / 2} .
$$

Although this is a common procedure in spectroscopy, distortion in the shape of the resonance curve occurs as a result of having only positive and finite integration limits. This is a common problem that under certain circumstances can be overcome. A good discussion of a solution used in nuclear magnetic resonance (NMR) is given by Farrar and Becker. ${ }^{16}$ If the phase of the driving signal can be mathematically (or physically) shifted by an angle $\phi$ until $f(t) \cos \left(2 \pi F t+\theta_{S}-\theta_{\mathrm{IF}}\right)$ is an even function, then $S(f)$ may be neglected because $f(t) \sin \left(2 \pi F t+\theta_{S}-\theta_{\mathrm{IF}}\right)$ will be odd and the integral of an odd function from $-\infty$ to $\infty$ is zero. The resulting $C(F)$ is then taken as a representation of the true resonant line shape. There are a number of techniques that work well in NMR for determining the angle $\phi$ for the phase correction. Unfortunately $\theta_{r}$ is a strong function of frequency in a weakly coupled system, such as ours, and must be measured or calculated at each frequency in order to make a proper phase correction. A good method for accomplishing this is not known. However, the amplitude spectrum is quite adequate for most studies especially when the major interest is in the center frequency of the resonances.

\section{REDUCTION OF MEASUREMENT ERRORS}

In the process of developing the measurement procedures and apparatus, we systematically identified the principal sources of measurement error. We also developed specific calibration and error-correction approaches needed to cope with such errors. Some of the measurement errors are caused by the inherent physical limitations of the measurement apparatus and signal-processing methods. Others were caused by factors such as sample shape and composition.

The physical origins of many of the measurement errors and methods for their elimination are identical to those we identified in previous publications. For example, dc offsets and drifts in the circuitry following the phasesensitive detectors are effectively eliminated by periodically 


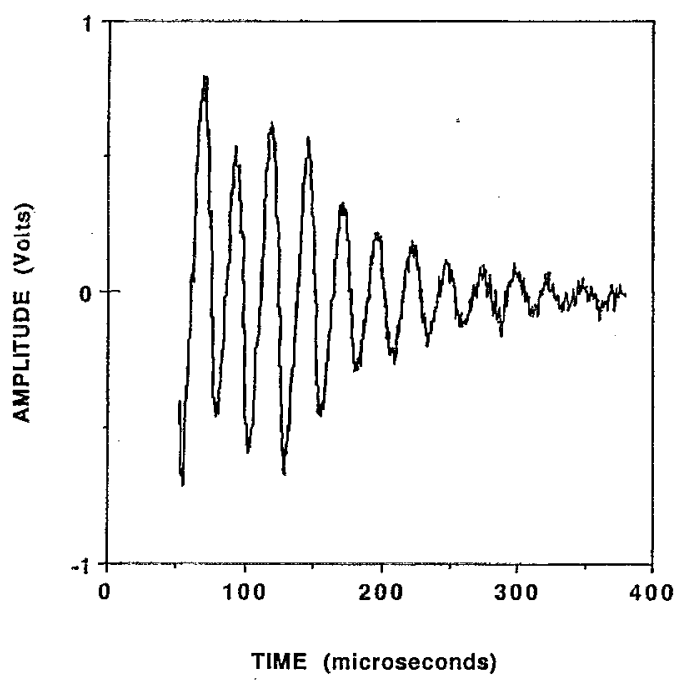

FIG. 4. Oscilloscope trace showing the filtered output of one of the phase sensitive detectors (multiplier 3 ). The excitation frequency is off resonance by approximately $38 \mathrm{kHz}$.

reversing the electrical phases of the in-phase and quadrature if reference signals applied to the analog multipliers 3 and 4. The effects of rf and IF leakage signals can be reduced by calibration. ${ }^{17}$

Reference 17 also describes procedures for dealing with the effects of unequal voltage gains of the two channels used in the phase-sensitive detector. Although the individual electrical components in these channels are carefully matched and adjusted, as needed, small variations are inevitable. The same is true of amplitude and phase errors caused by the lack of a perfect quadrature relationship between the two IF reference signals applied to analog multipliers 3 and 4.

The dominant source of measurement error in resonance experiments, which employ weakly coupled ultrasonic transducers such as the EMAT, is the leakage of cw signals generated either by the IF oscillator or the transmitter circuitry. The leakage in the transmitter is caused by the less-than-perfect Off/On ratio of the gated rf amplifier. Also, the open-coil design of an EMAT makes it particularly sensitive to magnetic and electric fields generated by

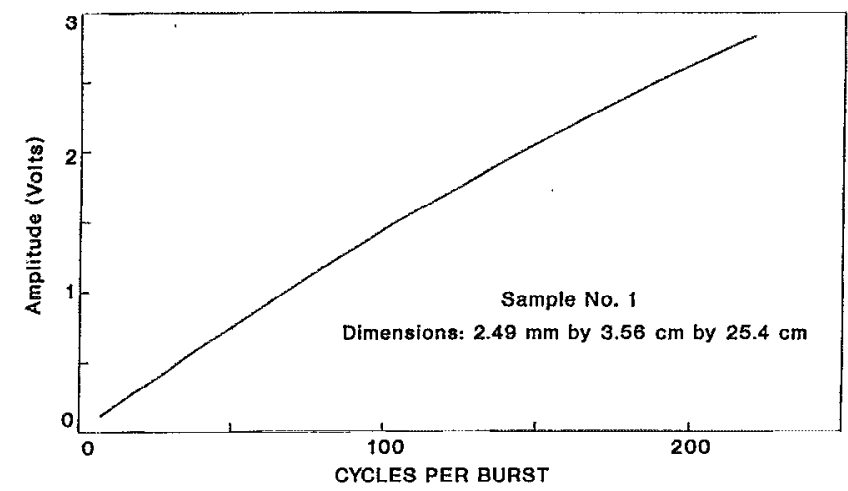

FIG. 5. The relationship between acoustic ringing amplitude (at resonance) and rf burst width in an aluminum plate. The frequency was set on the third harmonic (approximately $1.9 \mathrm{MHz}$ ).

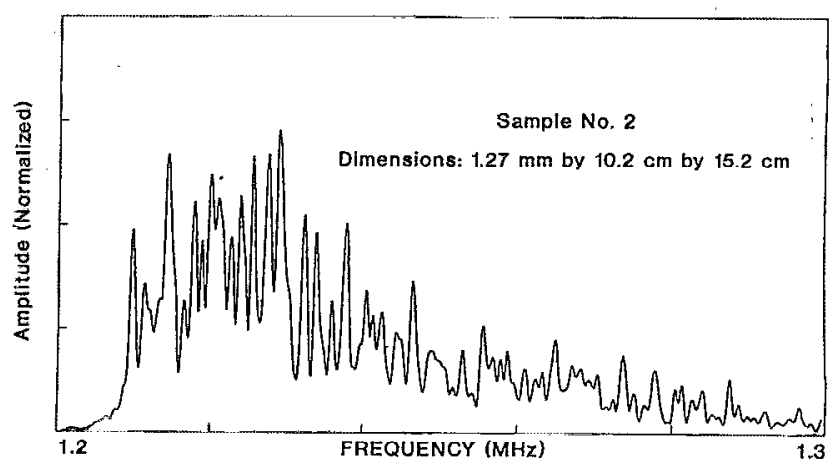

FIG. 6. Undamped fundamental $(N=1)$ resonance in an aluminum plate (sample 2).

other external sources. Such sources typically include distant radio stations, electrical lighting, and other instruments in the laboratory. If the interfering signals are stable in time, then they can be compensated by using appropriate calibration procedures or removed by averaging.

Considerable care must be exercised to ensure that all aspects of the acoustic and electrical environment, other than that of the sample, are stationary. Experimentally, we found that the amplitude and phase of the unwanted leakage signals are dependent on the geometrical and electrical characteristics of the samples used for calibrating the apparatus. For best results, the geometrical and electrical characteristics of calibration specimens should be similar to those of the resonant samples.

\section{EXPERIMENTAL RESULTS}

To illustrate the technique, signals were first obtained using two different aluminum-alloy sheets. The composition and metallurgical history of the materials were largely unknown. However, the signals are representative of other samples now under study. ${ }^{18}$

While performing measurements, it is convenient to observe the filtered output of multipliers 3 or 4 on an oscilloscope. The corresponding time wave form is shown in Fig. 4. Both the "ring-down" time and the signal-to-noise ratio of the received signals are generally functions of the acoustic attenuation in the sample. The low-order resonances in a low-loss material such as aluminum may exhibit very long ring-down times. The effects of weak coupling of the EMAT and low attenuation in the sample can be observed by monitoring the signal strength as a function of the received if burst width. This also is done in Fig. 5, where the signal amplitude continues to increase even at a burst width of 255 cycles. This phenomenon may become the basis for attenuation measurements at a single frequency.

Very long ring-down times may pose signal-processing difficulties. A typical frequency scan is illustrated in Fig. 6. A number of independent effects such as reflections from the edges of the plate contribute to the complicated appearance of the scan. Mode conversions may also be involved. In order to mitigate such effects, we used silicone rubber to 


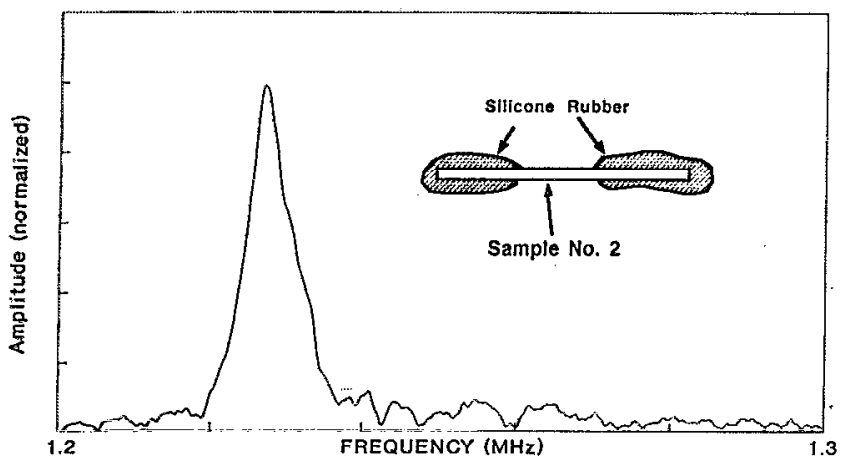

FIG. 7. Damped fundamental rcsonance in sample 2.

damp vibrations in the edges and surfaces of the specimen surrounding the EMAT. The effects of the damping are shown in Fig. 7.

Typical spectra resulting from a shear-wave birefringence experiment are shown in Fig. 8. In this case, the sample dimensions are $2.49 \mathrm{~mm} \times 35.6 \mathrm{~mm} \times 254 \mathrm{~mm}$ and the signals were projected along the long dimension. The shear-wave birefringence responsible for the splitting of the resonant frequency results from the metallurgical texture introduced in fabrication. ${ }^{15-17}$ The resonant frequencies of the first 26 orders of a shear mode polarized along the long direction are depicted in Fig. 9.

The resonant frequencies are related to the acoustic velocity by

$$
F=\frac{N V}{2 d},
$$

where $d$ is the sample thickness, $V$ is the velocity, and $N$ is the harmonic number. In order to test the resolution capability of the method, both longitudinal and shear-wave resonances were measured repeatedly in a plate of aluminum alloy $1.2 \mathrm{~mm}$ thick. The resonant frequencies were

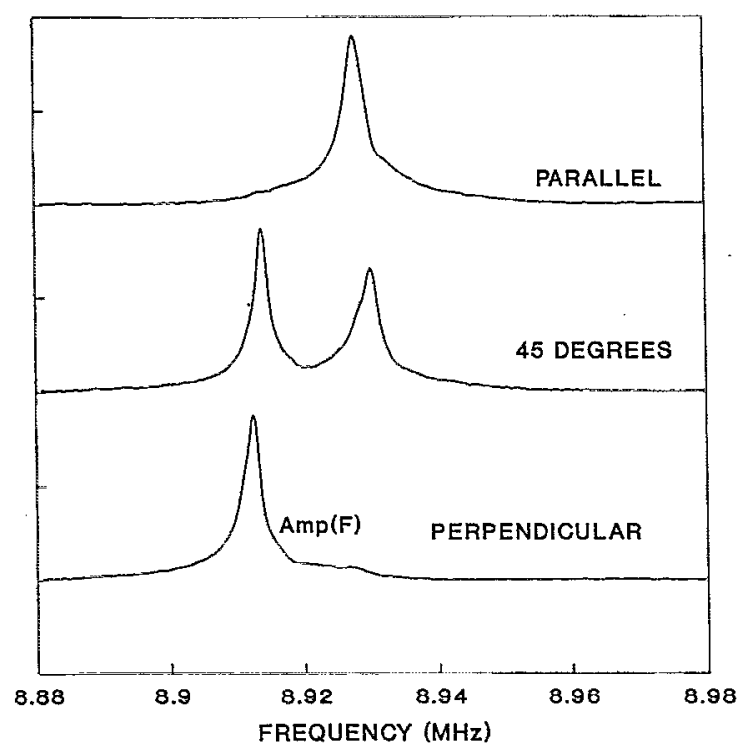

FIG. 8. The effects of shear-wave polarization direction for the 15 th harmonic $(N=15)$ in sample 1 . Signal amplitude has been normalized.

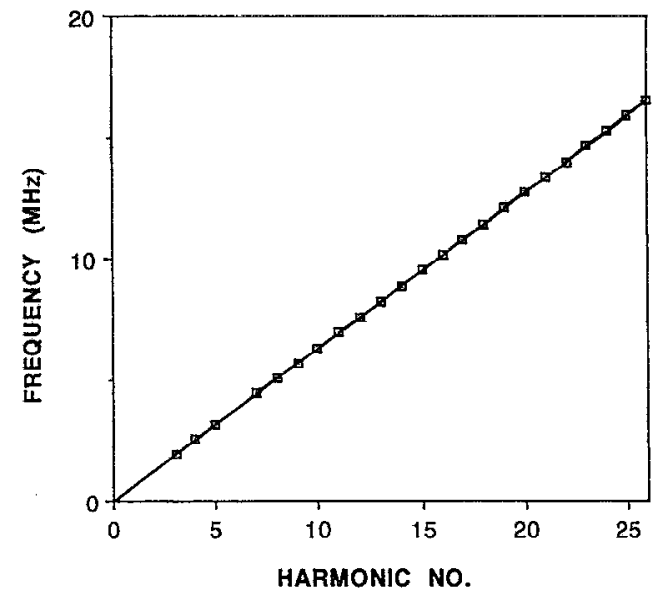

FIG. 9. Resonant frequency measurements up to $N=26$ for sample 1 . Measurements were made for shear waves polarized perpendicular to the long axis of the plate.

obtained by fitting a Gaussian curve to each peak and reading the center axis. The following are typical results:

Case 1: 4th longitudinal resonance using 10-mm-diam EMAT:

Average frequency $=10.48788 \mathrm{MHz}$,

Standard deviation $=36 \mathrm{~Hz}$,

Velocity $=6.2927 \times 105 \mathrm{~cm} / \mathrm{s}$.

Case 1: 6 th shear resonance using a $5 \times 6 \mathrm{~mm}$ diam EMAT:

Average frequency $=7.710711 \mathrm{MHz}$,

Standard deviation $=15.2 \mathrm{~Hz}$,

Velocity $=3.0843 \times 10^{5} \mathrm{~cm} / \mathrm{s}$.

Case 1: 4 th shear resonance using a $14 \times 20 \mathrm{~mm}$ diam EMAT:

Average frequency $=5.172521 \mathrm{MHz}$,

Standard deviation $=32 \mathrm{~Hz}$,

Velocity $=3.10351 \times 10^{5} \mathrm{~cm} / \mathrm{s}$.

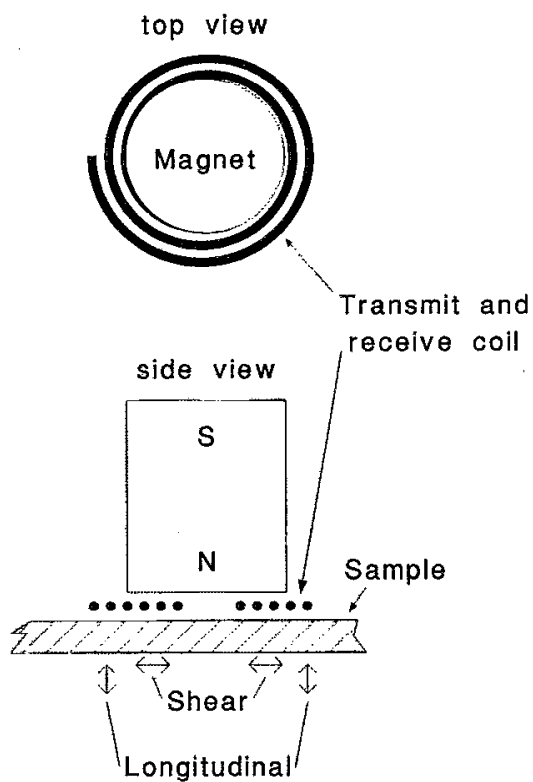

FIG. 10. Diagrammatic representation of an EMAT which produces both shear and longitudinal waves. 
The resolution of these measurements is of the order of a few parts per million which is better than other pulsed methods in solids, especially in thin samples. ${ }^{6}$ The accuracy of the method should also be quite good because the effect of the weakly coupled transducer (EMAT) on the resonant frequency is minimal. The velocity calculated from Eq. (12) for the 4th shear resonance is about $0.6 \%$ higher than the velocity for the 6 th shear resonance. This is not due to the different EMATs but to a different position on the sample. The thickness, texture, parallelness, and residual stress will all vary from point to point on samples of this type. Although the longitudinal and shear-wave resonances reported here were measured with separate transducers, the simultaneous monitoring of both orthogonally polarized shear wave resonances recently has been shown to be useful, not only in texture determination, but also in measuring stress in thin plates. ${ }^{18}$ This measurement has been facilitated by EMAT designs that generate both longitudinal waves and radially polarized shear waves. An example of such an EMAT is shown in Fig. 10.

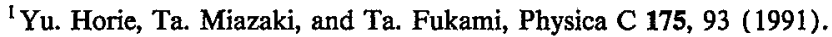

${ }^{2}$ H. J. McSkimmin, in Physical Acoustics, edited by W. P. Mason (Academic, New York, 1964), Vol. IA, pp. 27-417.
${ }^{3}$ D. I. Bolef and J. G. Miller, in Physical Acoustics, edited by W. P. Mason (Academic, New York, 1971), Vol. VIII, pp. 95-199.

${ }^{4}$ J. G. Miller and D. I. Bolef, J. Appl. Phys. 41, 2282 (1970).

${ }^{5}$ J. G. Miller, J. Acoust. Soc. Am. 53, 710 (1973).

${ }^{6}$ M. A. Breazeale, John H. Cantrell, Jr., and J. S. Heyman, in Methods of Experimental Physics, edited by P. D. Edmonds (Academic, New York, 1981), pp. 67-135.

${ }^{7}$ T. Pialucha, C. C. H. Guyott, and P. Cawley, Ultrasonics 27,270 (1989).

${ }^{8}$ G. Maze, J. L. Izbicki, and J. Ripoche, J. Acoust. Soc. Am. 77, 1352 (1985).

${ }^{9}$ K. Kawashima and O. B. Wright, J. Appl. Phys. 72, 4830 (1992).

${ }^{10}$ A. I. Kondratev, Sov. Phys. Acoust. 36, 262 (1990).

${ }^{11}$ M. Luukkala, S. P. Heikilla, and J. Surakka, Ultrasonics 9, 201 (1971).

${ }^{12}$ M. Luukkala and P. Merilainen, Ultrasonics 11, 218 (1973).

${ }^{13}$ A. V. Clark, Y. Berlinsky, N. Izworski, Y. Cohen, D. V. Mitrakovic, and S. R. Schaps, Res. Nondest. Eval. 4, 165 (1992).

${ }^{14}$ W. G. Clark, Rev. Sci. Instrum. 35, 316 (1964).

${ }^{15}$ A. Avogadro, G. Bonera, and M. Villa, J. Magn. Reson. 35, 387 (1979).

${ }^{16}$ Farrar and Becker, Pulse and Fourier Transform NMR (Academic, New York, 1971), Chap. 5.

${ }^{17}$ C. M. Fortunko, G. L. Petersen, B. B. Chick, M. C. Renken, and A. L. Price, Rev. Sci. Instrum. 63, 3477 (1992).

${ }^{18}$ H. Fukuoka, M. Hirao, T. Yamasaki, H. Ogi, G. L. Petersen, and C. M. Fortunko, in Review of Progress in Quantitative Nondestructive Evaluation, edited by D. O. Thompson and D. E. Chimenti (Plenum, New York, 1993), Vol. 12, pp. 2129-2136. 